\title{
Quiescent chromospheric response to the (E)UV/Optical flare radiation field on dMe stars
}

\author{
E.R. Houdebine ${ }^{1}$ and C.J. Butler ${ }^{2}$ \\ 1 Institut d'Astrophysique Spatiale, Univ. d'Orsay, bt. 120, 91400 Orsay, France. \\ ${ }^{2}$ Armagh Observatory, Armagh, BT61 9DG, N. Ireland.
}

\begin{abstract}
We examine the response of the quiescent chromosphere to the large (E)UV and optical continuum and spectral line radiation field arising from a flare. We show that during a UV Ceti type flare, which displays a large U-band enhancement, a major part of the Balmer line flux may arise from the "quiescent chromosphere", rather than the heated flare plasma itself. This leads us to distinguish two main phases in the Balmer lines, as first proposed by Houdebine et al. (1989): an early, mainly impulsive, phase, driven by radiative pumping of the quiescent chromosphere, which is mainly correlated with continuum variations, and a later one, related to the thermal flare phase, which arises from the cooling of the flare plasma itself. The effect of the radiative pumping is much larger for stellar ( $\mathrm{dMe}$ ) than for solar flares, due to substantial differences in the flare, relative to the quiescent, level and the quiescent chromospheric density and temperature.
\end{abstract}

\section{.1 Introduction}

Several detailed studies have been carried out on the soft X-ray backwarming from flares but, so far, none dealing with the chromospheric changes due to optical and (E)UV enhancements. The observed U-band variations - up to 5 magnitudes during a stellar flare drastically changes the ionisation equilibrium of Hydrogen and other elements.

\section{.2 Lyman, Balmer and Paschen photo-ionisation and photo-excitation}

In order to estimate, qualitatively, the effect of the flare radiation field on the quiescent chromosphere, we compare the additional net radiative rates produced by the flare radiation to the net rates for the quiet state. The quiescent chromosphere from which we computed the rates has been presented by Houdebine and Panagi (1989) (16 levels in NLTE, 70 lines and 9 continua). Then, to compare their effects, we considered a local increase of ten times the quiescent level, (equivalent to a $1.4 \mathrm{mag}$. U-band flare), in the Lyman, Balmer and Paschen continua. We computed the net photoionisation rates produced by such a radiation field at several levels in the atmosphere: at the temperature minimum, and at the Balmer and Lyman line formation regions. These rates are given in Figures 1a,b for two regions, as the $b$ - flare rates, while the atomic net rates in the quiet state are labelled as $b-b$ and $b-f$. 
From this diagram, it is striking that even small enhancements of the Lyman continuum will have a large impact on the Hydrogen ionisation equilibrium, at any atmospheric level, as the flare rate is at least 20 times larger than any other net rate.

Conversely, the same relative increase in the Paschen continuum $(8210 \AA$, between the Johnson $\mathrm{R}$ and $\mathrm{I}$ bands) creates a rather small rate, such that the relatively weak enhancement here during flares has little effect on the quiescent chromosphere.

On the other hand, such an increase of the Balmer continuum introduces a rate of the order of, or larger than, the rates in the quiescent state. Furthermore, the local Balmer continuum strength depends on the distance from the flare site, and for a 5 magnitude U-band flare, it will, in the vicinity of the flare, be $\sim 2000$ times the quiescent level, (i.e. 200 times that above). Such large variations drastically and instantaneously change the Hydrogen ionisation equilibrium throughout the surrounding quiescent chromosphere with the amplitude of the effect related to the magnitude and duration of the flare. An increase of the Lyman continuum flux has the most important effect on the atomic equilibrium, but its penetration in the quiescent chromosphere is limited by the large opacity of the chromospheric plasma. Conversely, although its effects are weaker, the Balmer continuum penetration is much larger. The main results are an increase of the Hydrogen ionisation fraction and local electron density, simultaneously with the depletion of the level 1 and 2 populations. This implies increased photo-recombination rates, decreased opacities of the medium in the Lyman and Balmer series, and thus an increase in the Balmer line emission from the surrounding "quiescent" chromosphere.

Therefore, we are led to distinguish two main phases for the evolution of Balmer line fluxes with time, (Houdebine et al. 1989); a phase driven by radiative phenomena and most often impulsive, and a second gradual one related to the thermal phase of the flare plasma. We note that no restriction is proposed for the relative strength or timing of these two phases; they can be totally blended or one of them can be so weak that it becomes negligible. The only constraint is that a large $\mathrm{U}$-band increase should be acompanied by a subsequent Balmer line increase.

An important point that one has to take into consideration, is the region where the flare radiant energy originates. If the flare continuum radiation arises from the photosphere, as proposed by de Jager et al (1989), the effects of the Lyman continuum will probably be confined within a very small volume around the flare site, due to the large chromospheric opacity. This also applies to the Balmer continuum, but to a lesser extent. Therefore, we can state that in that case, the flare will affect the upper chromosphere down to deeper chromospheric layers, but with a limited lateral extent. Conversely, if the radiation arises mostly from the low corona or high chromospheric levels, as in the solar two-ribbon white light flares, a surprisingly large chromospheric region is subjected to substantial changes, particularly the external layers where the Lyman and Balmer lines are formed.

\section{.3 Correlation between U-band enhancements and Balmer line profiles and fluxes}

Although no observations have been made of the Lyman continuum during stellar flares, the solar analogy as well as IUE stellar flare observations (Butler et al. 1981, Bromage et al. 1986) lead us to expect large and impulsive variations. 
We saw that the enhancements in the Balmer and Lyman continua should increase the quiescent chromospheric emission in the Balmer lines. Thus, the rather good correlation between the U-band and Balmer lines enhancements, (see Butler, 1988), may be due to Balmer and Lyman continuum variations.

To illustrate this point, we report to Houdebine et al. (1990) and Rodonò et al. (1989) who give respectively the continuum and normalised line flux time-profiles, and the U-band variations, during the AD Leo flare on the 28 March 1989 at 3:21 UT (see also Houdebine et al. 1989). The continuum and U-band impulsive changes are followed by a similar behaviour in the Balmer lines, with the time delay observed in the lines in agreement with the mean recombination time of electrons at chromospheric densities. Similarly, Doyle et al. (1988) found a good time correlation during flares on $\mathrm{YZ} \mathrm{CMi.}$

The flare on $\mathrm{AD}$ Leo displays two rather distinct Balmer line phases; the former more impulsive than the latter. During the former phase, the line profiles of the Balmer lines show two very different components, (Figure 2). A two Gaussian fit gives FWHM values of $\sim 4.3$ and $\sim 15.3 \AA$ respectively, with fluxes of $\sim 2700$ and $\sim 1100010^{-16} \mathrm{ergs} / \mathrm{cm}^{2} / \mathrm{s}$. We interpret these interesting features, as being respectively the signature of a highly turbulent flare plasma, and the quiescent chromospheric reponse to the large flare radiation field.

In order to confirm the previous suggestions, we computed the effect of a Balmer continuum backwarming, arising from the top of the chromosphere, with a modified version of the Carlsson and Sharmer NLTE-Radiative-Transfer code (see Scharmer and Carlsson 1985 for the original version). A few runs have been performed that confirm the previous discussion. We show in Figure 3 the $H_{\gamma}$ profiles of the quiescent chromosphere, and of the quiescent chromosphere irradiated by an increased Balmer continuum. These computations show that the flux has notably increased for all the Balmer series. Detailed results will be published later.

\section{References}

Bromage et al., $M N R A S, 220,1021$.

Butler, C.J., Byrne, P.B., Andrews, A.D., Doyle, J.G.: MNRAS, 197, 815 (1981)

Butler, C.J., Rodono, M., Foing, B.H., Haisch, B.M.: Nature, 321, 679 (1986)

Butler, C.J.: IrishAstr.J. 18, 198 (1988)

De Jager et al.: Astron. and Astrophys., 211, 157 (1989)

Doyle, J.G., Butler, C.J., Byrne, P.B., Van den Oord, G.H.J.: Astron. Astrophys., 193, 229 (1988)

Hawley, L.S.: IAU Coll. on Solar and Stellar Flares, Poster Papers, Catania Astrophys. Obs. Special Volume, Eds. Haisch and Rodono (1989)

Houdebine, E.R., Butler, C.J., Panagi, P.M., Rodono, M., Foing, B.H.: IAU coll. 104 on Solar and Stellar Flares, Poster Papers, Eds. Haisch and Rodono, (1989)

Houdebine, E.R., Butler, C.J., Panagi, P.M., Rodono, M., Foing, B.H.: Submitted to Astron. Astrophys., (1990)

Houdebine, E.R., Panagi, P.M.: To appear in Astron. Astrophys., (1989)

Houdebine, E.R., Butler, C.J., Panagi, P.M., Rodono, M., Foing, B.H.: submitted to Astron. and Astrophys., (1989)

Mullan, D.J., Tarter, C.B.: Ap. J., 212, 179 (1977)

Rodono, M., Houdebine, E.R., Catalano, S., Foing, B.H., Butler, C.J., Scaltriti, F., Cutispoto, G., Gary, D.E., Gibson, D.M., and Haisch, B.M.: IAU coll. 104 on Solar and Stellar Flares Poster Papers, Eds. Haisch and Rodono, (1989)

Scharmer, G.B, Carlsson, M.: J. Comp. Phys., 59, 56 (1985) 

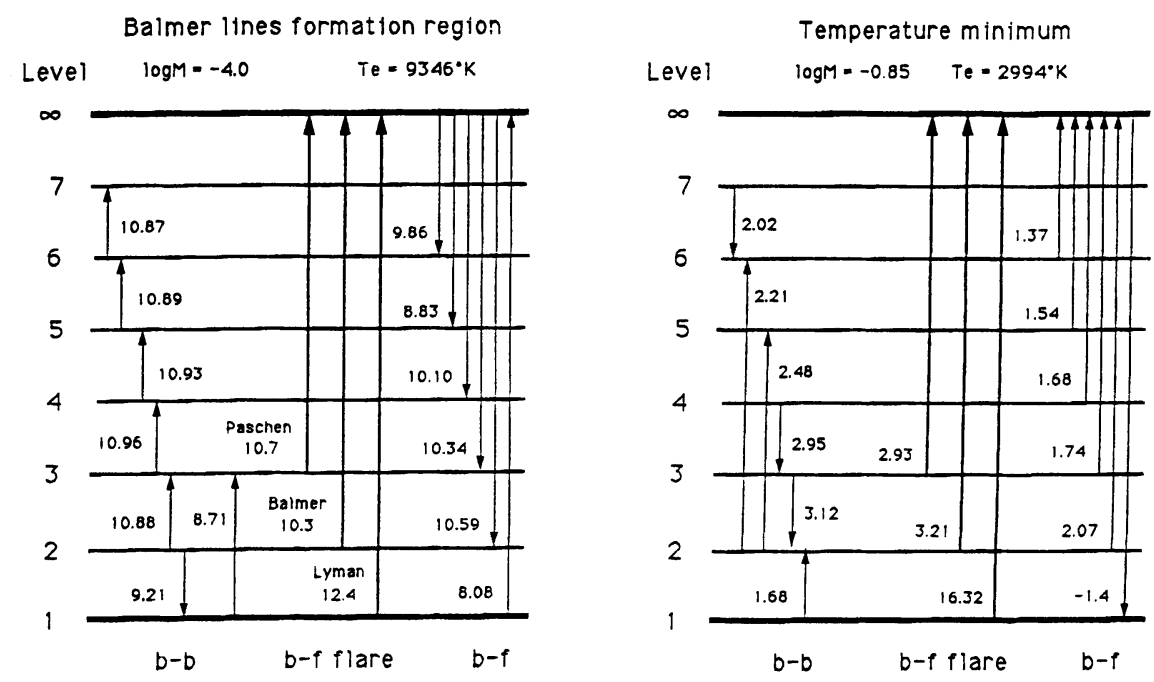

Fig. 1a, 1b: The logarithm of the main rates between the Hydrogen levels are given on the right and left sides (b-f and $\mathrm{f}-\mathrm{f}$ ). The values given as $b$ - $f$ flare, are the rates that would be created by an increase of the local photoionisation continua equal to, ten times the stellar quiescent flux. The amazingly high amplitudes of such flare induced rates imply a large atomic equilibrium rearrangement in the quiescent chromosphere (see text).

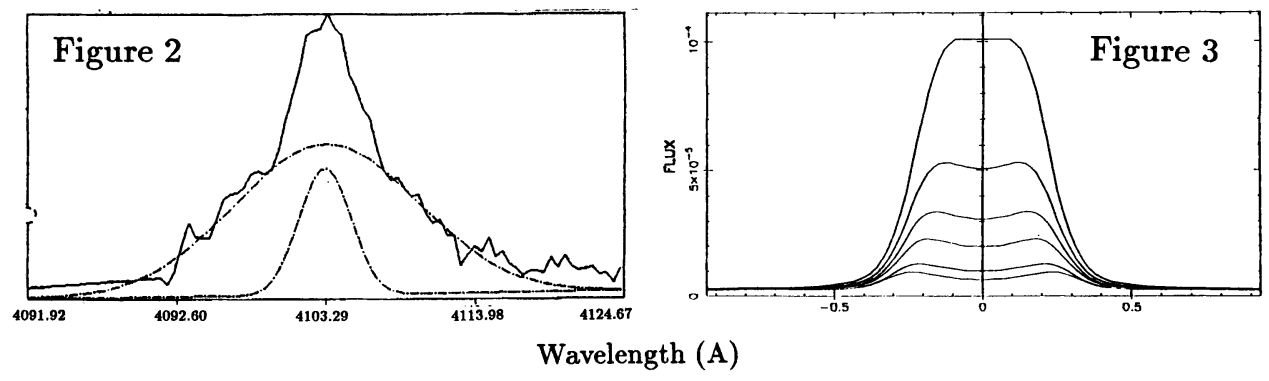

Fig. 2: The $H_{\gamma}$ line profile during the first spectrum of the AD Leo flare (28 March 1984, 3:21 UT). The profile displays two main components that here are fitted with two Gaussians. The narrower component is interpreted as being the signature of the quiescent chromospheric response to the flare radiation field.

Fig. 3: $H_{\gamma}$ profiles, for the quiescent chromosphere, and the quiescent chromosphere under a simulated flare radiation field (only the Balmer continuum). The enhancement in the Balmer continuum ( $\sim \mathrm{U}$-band) relative to the quiescent stellar level was respectively, 1.0, 1.95, 5.13, 8.91, 16.2 and 38.0, with the lower profile the quiescent emission. 